\title{
Ocular hypotensive efficacy and safety of once daily carteolol alginate
}

\author{
Philippe Demailly, Catherine Allaire, Claude Trinquand, for the Once-daily Carteolol \\ Study Group
}

exhibits a viscosity low enough to be compatible with topical administration without blurring effect. Sodium alginate is a natural polymer product with bioadhesive properties, ${ }^{6}$ used in many pharmaceutical preparations including those for reflux oesophagitis. ${ }^{7}$ In animals, an alginate formulation of carteolol $1 \%$ and $2 \%$ provides good intraocular delivery of carteolol, enhanced relative to standard solution. ${ }^{8}$ In a water loaded rabbit model of aqueous humour dynamics, the alginate formulation of carteolol had a longer duration of action than the standard solution. ${ }^{8}$ In a short term study in normal volunteers, the alginate formulation was found to be of similar comfort as the standard solution, with a possible longer duration of ocular hypotensive action.

The objective of this study was to evaluate the efficacy and safety of carteolol alginate administered once daily in comparison with standard carteolol solution administered twice daily in a long term study.

Results-At 0900 (presumed trough) on day 60, mean reductions in intraocular pressure (IOP) from baseline were 6.09 (SD 2.97) and 6.09 (3.18) $\mathrm{mm} \mathrm{Hg}$ for the standard carteolol and alginate, respectively. At 1100 (presumed peak), mean reductions were $6.51(2.53)$ and $6.47(2.76)$ mm Hg, respectively. Results were similar at other times (day 15 and day 120). The most common side effect was transient stinging on instillation of drops, which did not differ significantly between groups. There were no differences of note in other ocular or systemic signs or symptoms.

Conclusion-The new alginate formulation of carteolol $2 \%$ given once daily was as effective as standard carteolol $2 \%$ given twice daily with no meaningful differences regarding safety.

Hôpital St Joseph, Institut du Glaucome, Paris, France

P Demailly

Scientific and Medical Affairs Department, Laboratoire Chauvin, Montpellier, France

C Allaire

C Trinquand

Correspondence to:

Claude Trinquand, MD,

Laboratoire Chauvin,

Scientific and Medical

Affairs Department, Le

Millenaire, BP 1174

F-34009 Montpellier cedex,

France

ctrinqua@chauvingroup.com

Accepted for publication 6 March 2001

\section{Methods}

This was a double masked, randomised, multicentre study, comparing two parallel treatment groups. Enrolled were adult patients with open angle glaucoma or ocular hypertension in one or both eyes. Patients using ocular hypotensive medication were required to undergo a washout as follows: $\beta$ adrenoceptor antagonists or sympathomimetics (3 weeks); latanoprost (1 week), pilocarpine, apraclonidine, or topical or oral carbonic anhydrase inhibitors (72 hours). For entry into the study the "study" eye(s) was (were) required to have an unmedicated IOP $\geqslant$ 23 at 0900 or 1100 and $<32 \mathrm{~mm} \mathrm{Hg}$ at 0900 and 1100 . This study was reviewed by an institutional review board, and all patients provided written informed consent. Excluded from the study were patients with angle closure, congenital, secondary glaucoma, or advanced glaucoma; contact lens wear during the study; any intraocular infection or inflammation, ocular trauma, ocular surgery, or laser trabeculoplasty within the previous 3 months; previous intolerance to carteolol; or contraindications to the use of $\beta$ adrenoceptor antagonists (for example, asthma, chronic obstructive pulmonary disease, moderate to severe atrioventricular block unless a pacemaker was implanted, or bradycardia $<45 \mathrm{bpm}$, etc). Ocular corticosteroid use during the study was prohibited. Patients who were using systemic medications such as adrenergic hypotension agents were allowed to participate if the condition and dosing regimen were stable. Also excluded were pregnant and lactating women.

A baseline examination was conducted, including measurements of heart rate and ard twice daily to once daily, carteolol was formulated with alginic acid. Alginate solution 
Table 1 Demographics

\begin{tabular}{lllll}
\hline & Standard & Alginate & Total & $p$ Value \\
\hline $\begin{array}{l}\text { No } \\
\text { Age (years) }\end{array}$ & 115 & 120 & 235 & 0.845 \\
$\quad \begin{array}{l}\text { Mean (SD) } \\
\text { (Range) }\end{array}$ & $\begin{array}{l}60.36(13.52) \\
(17-85)\end{array}$ & $61.40(12.11)$ & $60.89(12.81)$ & \\
$\begin{array}{l}\text { Sex (n/\%) } \\
\quad \text { Male }\end{array}$ & $64(55.7 \%)$ & $72(60.0 \%)$ & $136(57.8 \%)$ & 0.500 \\
$\quad$ Female & $51(44.4 \%)$ & $48(40.0 \%)$ & $99(42.1 \%)$ & 0.222 \\
$\begin{array}{l}\text { Iris colour (n/\%) } \\
\quad \text { Blue-green-grey }\end{array}$ & $50(43.5 \%)$ & $53(44.2 \%)$ & $73(31.1 \%)$ & \\
$\quad$ Hazel & $34(29.6 \%)$ & $25(20.8 \%)$ & $59(25.1 \%)$ & \\
$\quad$ Brown & $31(30.0 \%)$ & $42(35.0 \%)$ & $73(31.1 \%)$ & 0.434 \\
$\quad \begin{array}{l}\text { Glaucoma diagnosis } \\
\quad \text { OAG }\end{array}$ & $71(61.7 \%)$ & $78(65.0 \%)$ & $149(63.4 \%)$ & $149(63.4 \%)$ \\
$\quad$ OHT & $44(38.3 \%)$ & $42(35.0 \%)$ & $86(36.6 \%)$ & $86(36.6 \%)$ \\
\hline
\end{tabular}

p Value for age by Wilcoxon two sample test (due to non-normal distribution), and for sex and iris colour by $\chi^{2}$. sum test depending on the distribution and ANOVA, and categorical measures were evaluated using the $\chi^{2}$ test (PROC TTEST, PROC NPAR 1 WAY, PROC GLM AND PROC FREQ, PC-SAS). A priori, the primary efficacy criterion was defined as the change from time relevant baseline IOP on day 60 , a period by which the IOP on the new treatment should have stabilised. Equivalence tests were carried out following the methodology described by Grouin and Coste. ${ }^{10}$ Two one sided $t$ tests were performed. If both $\mathrm{p}$ values were inferior to 0.05 , then equivalence between the two formulations was the conclusion.

\section{Results}

blood pressure, visual acuity, automated threshold visual field (if not performed within the previous 6 months), biomicroscopy, IOP measurement by Goldmann applanation at 0900 and 1100. After the last baseline IOP measurement, patients were assigned to receive, in a double masked fashion, either $2 \%$ carteolol alginate four times daily $(\sim 0900)$ or standard 2\% carteolol solution (Carteol, Laboratories Chauvin, Montpellier, France), twice daily ( 0900 and 2100). The randomisation code was prepared using the PROC RANUNI procedure (PC-SAS Version 6.12, SAS Institute, Cary, NC, USA) with a block size of four.

The composition of carteolol-alginate was identical to that of market carteolol, with the exception of the addition of $1 \%$ alginate. The formulations were identical in tonicity and viscosity. The $\mathrm{pH}$ of the solutions was similar. The vehicle for the carteolol-alginate group was formulated the same as the carteololalginate formulation, with the exception of carteolol. Patients used the medication in one or both eyes. The masking was maintained through the use of a vehicle in the evening for the alginate group. Patients were evaluated at baseline, 15, 60, and 120 days, with IOP measurements just before instillation of medication (0900) and 2 hours after in-office instillation of medication (1100). Slit lamp examinations were performed at each follow up examination, together with measurement of heart rate and blood pressure (1000) and ocular tolerance of the medication (1100). At day 120, visual acuity was assessed with the Snellen or decimal Monoyer scale, and visual fields were performed.

Statistical analysis of ocular hypotensive efficacy was performed on the eye with the higher IOP at a mean of the 0900 and 1100 baseline, or, if equal, the right eye. With a sample size of 105 patients per treatment group, this study was planned for $95 \%$ power to detect a difference in treatments on IOP of $2 \mathrm{~mm} \mathrm{Hg}$, assuming a standard deviation of $4 \mathrm{~mm} \mathrm{Hg}$, and a two tailed alpha of 0.05 . The study also had $80 \%$ power to detect a treatment difference of $1.5 \mathrm{~mm} \mathrm{Hg}$. An overenrolment of approximately $5 \%$ was planned to account for any premature withdrawals or disqualifications. Both intent to treat and per protocol analyses were planned. Continuous measures were analysed using $t$ test or Wilcoxon rank
Entered into the study were 235 patients (115 standard and 120 alginate), plus one subject who did not return after day 0 . In the standard group, five patients did not complete the study: lost to follow up (one), adverse events (two, dizziness, thorax pain and sweats of moderate intensity; and mild ocular irritation), worsening of disease (one), and cancellation of consent (one). In the alginate group a similar number of patients did not complete the trial: adverse events (three, tinnitus and watering of the eye; bradycardia; and dizziness - all judged mild or moderate), cancellation of consent (one), and poor compliance (one).

Patient demographics are shown in Table 1. The population was $58 \%$ male with a mean age of 61 years, with iris colour distributed among blue-green-grey, hazel, and brown irides. Most patients were white. Approximately two thirds of the patients were diagnosed with primary open angle glaucoma $(63.4 \%, 149 / 235)$, including three patients with pigmentary glaucoma (one in standard group and two in the alginate group). There were no statistically significant differences between treatment groups $(p \geqslant 0.22)$. Most $(87 \% ; 204 / 235)$ patients received treatment in both eyes. Also, most $(64 \%, 151 / 235)$ patients were using at least one systemic medication. Before study entry, $51 \%$ of patients $(120 / 235)$ were using an ocular hypotensive medication. For 102 of these patients, this medication was a topical $\beta$ adrenoceptor antagonist.

Mean intraocular pressure at 0900 and 1100 is shown in Figures 1 and 2 respectively, and mean change from baseline in intraocular pressure in Tables 2 and 3.

From a baseline of approximately $24-25 \mathrm{~mm}$ $\mathrm{Hg}$, mean reductions ranged from 5.5 to 6.5

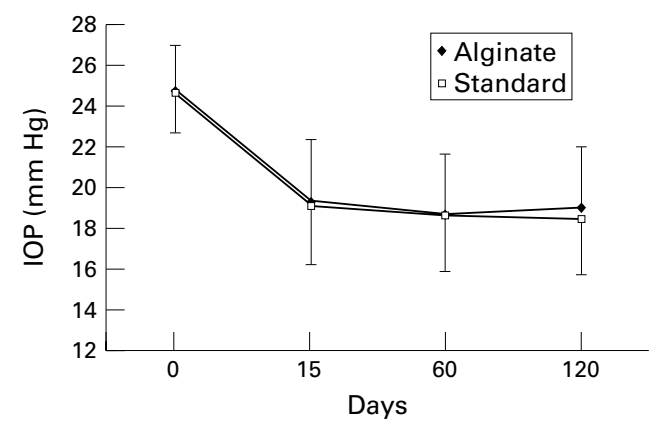

Figure 1 Mean intraocular pressure (IOP) at 0900 (SD). 


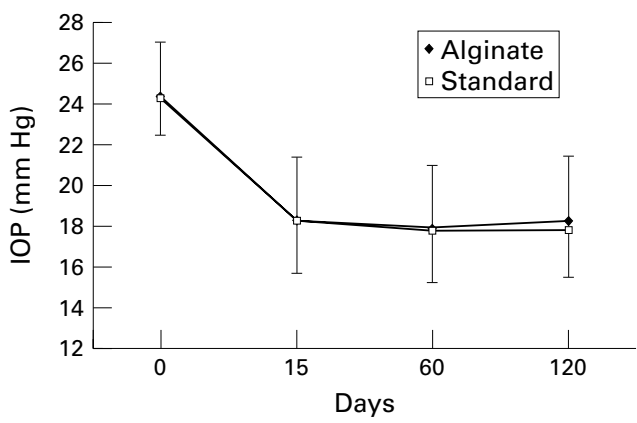

Figure 2 Mean intraocular pressure (IOP) at 1100 $(S D)$.

$\mathrm{mm}$ to an IOP of approximately $18 \mathrm{~mm} \mathrm{Hg}$ in both treatment groups. The ocular hypotensive efficacy of carteolol was slightly greater at 1100,2 hours after the last instillation, relative to 0900 , approximately 24 or 12 hours after the last instillation of alginate or standard, respectively. The IOP course was statistically significant within time (time effect: $p=0.0001$ in the repeated measures analysis). There was no evidence of drift with either treatment. In the equivalency analyses, all measures were within the plus or minus $2 \mathrm{~mm} \mathrm{Hg}$ interval for equivalence and the two unilateral $t$ tests were highly significant $(\mathrm{p}<0.005)$ at each time of evaluation.

A per protocol analysis was performed on the 210 patients (102 in standard and 108 in alginate groups) without major protocol violations. Results were similar to the intent to treat analysis. The confidence intervals were $(-0.57$, $+0.80)$ and $(-0.46,0.66) \mathrm{mm} \mathrm{Hg}$ for day 60 at 0900 and 1100 , respectively.

No change was noted in distance visual acuity for $78.3 \%(90 / 115)$ of standard treated patients and in $70.0 \%(84 / 120)$ of alginate treated patients, without any statistically significant difference between the two treatment groups $\left(\chi^{2}, \mathrm{p}=0.20\right)$. Similar results were seen with fellow eye.

At day 120, visual fields were unchanged for $78.3 \%(90 / 115)$ of standard carteolol treated

Table 2 Intraocular pressure at $0900(\mathrm{~mm} \mathrm{Hg}):$ mean (SD) change from day 0 baseline

\begin{tabular}{|c|c|c|c|c|c|c|c|c|}
\hline \multirow[b]{2}{*}{ Day } & \multicolumn{3}{|c|}{ Standard } & \multicolumn{3}{|c|}{ Alginate } & \multirow{2}{*}{\multicolumn{2}{|c|}{$90 \%$ CI }} \\
\hline & No & Mean & $S D$ & No & Mean & $S D$ & & \\
\hline 0 & 115 & 24.67 & 1.98 & 120 & 24.82 & 2.14 & & \\
\hline 15 & 115 & -5.58 & 3.13 & 120 & -5.50 & 2.99 & -0.74 & +0.57 \\
\hline 60 & 111 & -6.09 & 2.97 & 117 & -6.09 & 3.18 & -0.67 & +0.67 \\
\hline 120 & 110 & -6.25 & 3.03 & 115 & -5.86 & 2.79 & -1.03 & +0.25 \\
\hline
\end{tabular}

At each time of evaluation, the two unilateral $t$ tests were significant (both $\mathrm{p}$ values $<0.005$ ), showing the equivalence of both treatments.

Table 3 Intraocular pressure at $1100(\mathrm{~mm} \mathrm{Hg})$ : mean (SD) change from day 0 baseline

\begin{tabular}{|c|c|c|c|c|c|c|c|c|}
\hline \multirow[b]{2}{*}{ Day } & \multicolumn{3}{|c|}{ Standard } & \multicolumn{3}{|c|}{ Alginate } & \multirow{2}{*}{\multicolumn{2}{|c|}{$90 \% C I$}} \\
\hline & $\mathrm{No}$ & Mean & $S D$ & No & Mean & $S D$ & & \\
\hline U & 115 & 24.31 & 1.83 & 120 & 24.42 & 2.63 & & \\
\hline 15 & 113 & -6.07 & 2.27 & 119 & -6.06 & 2.72 & -0.60 & +0.57 \\
\hline 60 & 111 & -6.51 & 2.53 & 116 & -6.47 & 2.76 & -0.40 & +0.30 \\
\hline 120 & 115 & -6.47 & 2.40 & 115 & -6.18 & 2.84 & -0.63 & +0.53 \\
\hline
\end{tabular}

At each time of evaluation, the two unilateral $t$ tests were significant (both $\mathrm{p}$ values $<0.005$ ), showing the equivalence of both treatments.
Table 4 Number and percentage of patients with very good or good tolerance upon instillation

\begin{tabular}{lllll}
\hline & \multicolumn{3}{c}{ Standard } & \multicolumn{3}{c}{ Alginate } \\
\cline { 2 - 5 } Day & No & $\%$ & No & $\%$ \\
\hline 15 & 114 & 99.1 & 119 & 99.2 \\
60 & 112 & 100.0 & 116 & 99.2 \\
120 & 109 & 98.2 & 114 & 99.1 \\
\hline
\end{tabular}

patients and $67.5 \%(81 / 120)$ of alginate treated patients for the study eye $(\mathrm{p}=0.12$ by $\left.\chi^{2}\right)$.

Adverse events were reported by 59 patients. Fifteen adverse events were assessed as drug related. Among these, four affected the eye and adnexa: two in the standard group (one irritation and one worsening of dry eye) and two in the alginate group (one ocular irritation and one superficial punctate keratitis). Eleven nonophthalmic adverse events were assessed as drug related: five in the standard carteolol group and six in the alginate group. These adverse events were known possible $\beta$ blocker side effects.

Two serious adverse events were reported during the study: unilateral worsening of existing age related macular degeneration (standard group) and hospitalisation for repair of hiatal hernia (alginate group). Neither was judged as drug related.

Subjective tolerance upon instillation was judged good or very good by $99 \%$ of alginate patients and by $98 \%$ of standard patients evaluated on day 120 (Table 4). Discomfort was reported by approximately $10 \%$ to $15 \%$ of patients in each treatment group at each visit (Table 5). These reports were similar in incidence in each group. The most frequent discomfort in both groups was a stinging sensation which generally lasted for a few seconds or a few minutes. The blurred vision sensation was recorded in $3 / 120$ patients of the alginate group and $2 / 115$ patients of the standard group.

At baseline, mean heart rate, systolic and diastolic blood pressure was approximately 73-74 bpm, 141-144 $\mathrm{mm} \mathrm{Hg}$, and 81-82 mm $\mathrm{Hg}$, respectively. Slight decreases in means were observed at follow up visits (up to $6 \mathrm{bpm}$, $8 \mathrm{~mm} \mathrm{Hg}$, and $3 \mathrm{~mm} \mathrm{Hg}$, respectively). There were no statistically significant differences between treatment groups $(\mathrm{p}=0.607$ to 0.852).

\section{Discussion}

Evaluated at both peak and trough, at an initial, middle, and long term stage, the new alginate formulation once daily was equivalent in ocular hypotensive efficacy to the standard solution formulation given twice daily. While a vehicle control would have been desirable, it is not possible to use in a chronic glaucoma study for ethical reasons. The positive control, standard carteolol solution, was similarly effective in the present study (reduction of $\sim 6 \mathrm{~mm} \mathrm{Hg}$, or $\sim 25 \%$ ) to that observed in previous studies. ${ }^{2411}$ Thus, the efficacy of the positive control, and the high power of the study ( $95 \%$ to detect a 2 $\mathrm{mm} \mathrm{Hg}$ difference) supports the statement of equivalency. 
Table 5 Description of discomfort upon instillation

\begin{tabular}{llll}
\hline Day & Standard & Alginate & p Value $\left(\chi^{2}\right)$ \\
\hline 15 & $19(16.5 \%)$ & $14(11.7 \%)$ & 0.284 \\
Stinging & 15 & 6 & \\
Blurred vision & 0 & 2 & \\
Burning & 1 & 2 & 0.923 \\
Miscellaneous & 3 & 4 & \\
60 & $12(10.7 \%)$ & $13(11.1 \%)$ & \\
Stinging & 10 & 10 & 0.930 \\
Blurred vision & 2 & 1 & \\
Burning & 0 & 0 & \\
Miscellaneous & 0 & 2 & \\
120 & $11(9.9 \%)$ & $11(9.6 \%)$ & \\
Stinging & 6 & 2 & \\
Blurred vision & 0 & 0 & \\
Burning & 2 & 4 & \\
Miscellaneous & 3 & & \\
\hline
\end{tabular}

Miscellaneous reports included: bad taste, discomfort, dry eye, flow in throat, grittiness, irritation, visual problem, and watering.

Special attention is directed towards the 0900 hour measurement. This is $\sim 24$ hours after the last dose of carteolol alginate, and yet the mean IOP is similar to the standard solution given 12 hours previously.

The ocular tolerance of the alginate was good. Again, it was similar to the standard formulation, which itself has been reported to be well tolerated. ${ }^{12}{ }^{13}$ Thus, the finding of no substantial comfort problems is also a positive attribute of the alginate. In particular, the incidence of blurred vision (less than 3\% in each group) is much lower than what has been reported with timolol in gel forming solution. ${ }^{13}$

With regard to safety, there was no significant difference between both treatment groups for all studied safety variables.

Both treatment groups experienced a slight decrease in mean heart rate and blood pressure. The intrinsic sympathomimetic activity of carteolol could explain the relatively small impact of carteolol on the cardiovascular function: carteolol has been shown to cause less bradycardia than timolol at night. ${ }^{14}$ In our study, cardiovascular parameters were measured only once per visit, 1 hour after the morning instillation. Thus, it is not possible to discuss what occurred at other times for the 24 hours.

The relatively few adverse events were similar to those previously reported for this class of compounds. ${ }^{15} 16$

In conclusion, the new alginate formulation of carteolol $2 \%$ given once daily was as effective as standard carteolol given twice daily with no meaningful differences regarding safety.

Conflict of interest: Professor Demailly serves as a consultant to Laboratories Chauvin, but has no proprietary interest. Drs Allaire and Trinquand are employees of Laboratories Chauvin

The Once-daily Carteolol Study Group consisted of 23 French clinical centres and one Swiss clinical centre: Y Lachkar, MD, H Graciès, MD, Hôpital St Joseph, Paris (Study
Coordinator: $\mathrm{Pr} \mathrm{Ph}$ Demailly); J P Adenis, $\mathrm{MD}, \mathrm{Ph}$ Bertin, $\mathrm{MD}$, CHRU Dupuytren, Limoges; JC Dascotte, MD, Loos; J
Flament, MD, J Szwarcberg, MD, Hospices Civils, Strasbourg; T Hoang-Xuan, MD, N Belayachi, MD, Hôpital Bichat, Paris; G Kretz, MD, Paris; MJ Le Rebeller, MD, Ph Cousin, MD, GH Pellegrin-Tripode, Bordeaux; G Lesieur, MD, Albi; M Montard, MD, F Majo, MD, CHR Jean Minjoz, Besançon; A Raspiller, MD, L Mala, MD, X Portrat, MD, Hôpital Central, Nancy; Ph Renard, MD, P Delacour, MD, Institut Arthur Vernes, Paris; D Sirbat, MD, Strasbourg; C Bouat, MD, S Vitte, MD, JP Ghipponi, MD, Hôpital Militaire Laveran, Marseille; L Pernod, MD, P Héliès, MD, CHA René Le Bas, Cherbourg; Pernod, MD, P Hectieng; C Rousse, MD, Chopin, MD, Hopital Louis Pasteur, Dôle; JL George, MD, P Lesure, MD, Hopital de Brabois, Vandoeuvreles-Nancy; J Ferraton, MD, Clinique Charcot, Ste-Foy-lesLyon; A Brézin, MD, ORivoal, MD, A Lefrançois, MD, Hôpital Cochin, Paris; G Arrouas, MD, Dijon; JD Grange, MD, L Kodjikian Hôpital de la Croix Rousse, Lyon; JL Kovalski, MD, Y Dordain MD, R Macarez MD, D Salabert MD, HIA "Clermont-Tonnerre", Brest; P Daubas MD, G Filliard MD, Hôpital Sainte-Anne, Toulon; AGarrec, MD, Polyclinique Sévigné, Cesson-Sevigné; A Mermoud, MD, F Achache MD, A Chabari MD, Hôpital Jules Gonin, Lausanne (Suisse).

Statistician: Alice Huntsman, $\mathrm{PhD}$, Clinica and Statistica (Issy les Moulineaux, France).

Coordinating centre: Catherine Allaire, MD, Sandrine George, MS, Anne Combe, MS, Florence Bernard, MS, Ghéziel El Hamdi, PhD, Scientific and Medical Affairs Department, Laboratoire Chauvin, Montpellier, France.

Medical writer: Gary D Novack, PhD (San Rafael, CA, USA).

1 Kitazawa Y, Azuma I, Takase M, et al. Ocular hypotensive effects of carteolol hydrochloride in primary open-angle glaucoma and ocular hypertensive patients. A doublemasked cross-over study for the determination of concentrations optimal for clinical use. Acta Soc Ophthalmol fpn 1981;85:798-804.

2 Keates EU, Friedland BR, Stewart RH, et al. Carteolol hydrochloride: controlled evaluations of its ocular hypotensive efficacy relative to its vehicle, and, in combination with pilocarpine, relative to timolol. F Glaucoma 1993;3:315-22.

3 pilocarpine, relative to timolol. F Glaucoma 1993;3:315-22. Stewart WC, Shields MB, Allen RC, et al. A 3-month com-
parison of $1 \%$ and $2 \%$ carteolol and $0.5 \%$ timolol in openangle glaucoma. Graefes Arch Clin Exp Ophthalmol 1991; 229:258-61.

4 Flammer J, Kitazawa Y, Bonomi L, et al. Influence of carteolol and timolol on IOP an visual fields in glaucoma: a multi-center, double-masked, prospective study. Eur $\mathcal{f}$ Ophthalmol 1992;2:169-74.

5 Blackwell B. Treatment adherence. Br f Psychiatry 1976; 129:513-31.

6 Evans LV. Mucilaginous substances from macroalgae: an overview. Symp Soc Exp Biol 1989;43:455-61.

7 Washington N, Steele RJ, Jackson SJ, et al. Patterns of food and acid reflux in patients with low-grade oesophagitisthe role of an anti-reflux agent. Aliment Pharmacol Ther 1998; 12:53-8.

8 Séchoy O, Tissié G, Sébastian C, et al. A new long acting ophthalmic formulation of carteolol containing alginic acid. Int f Pharm 2000;207:109-16

9 Rosenlund EF. The intraocular pressure lowering effect of timolol in gel-forming solution. Acta Ophthalmol Scand 1996;74:160-2.

10 Grouin JM, Coste M. Essais d'équivalence: méthodologie et analyse. In: Bouvenot G, Vray M. Essais cliniques: théorie,
pratique et critique. 3rd ed. Paris: Flammarion, 1999:67-81.

pratique et critique. 3rd ed. Paris: Flammarion, 1999:67-81.
11 Stewart WC. Carteolol, an ophthalmic $\beta$-adrenergic blocker with intrinsic sympathomimetic activity. F Glaucoma 1994; 3:339-45

12 Scoville B, Krieglstein GK, Then E, et al. Measuring druginduced eye irritation: a simple new clinical assay. 7 Clin Pharmacol 1985:25:210-18.

13 Scoville B, Mueller B, White BG, et al. A double-masked comparison of carteolol and timolol in ocular hypertension. Am 7 Ophthalmol 1988;105:150-4.

14 Netland PA, Weiss HS, Stewart WC, et al. Cardiovascular effects of topical carteolol hydrochloride and timolol maleate in patients with ocular hypertension and primary maleate in patients with ocular hypertension and primary

15 Novack GD, Leopold IH. The toxicity of topical ophthalmic beta-blockers. F Toxicol Cutan Ocul Toxicol 1987;6:283-97.

16 Akingbehin T, Sunder Raj P. Ophthalmic topical beta blockers: review of ocular and systemic adverse effects. $\mathcal{F}$ Toxicol Cutan Ocul Toxicol 1990;9:131-47. 


\section{LETTER TO THE EDITOR}

Analysis of publication trends in two internationally renowned ophthalmology journals

EDITOR,-International journals represent a forum for exchange of current information with contributions from all over the world. High standards are essential. In this report, we compared the publishing trends of two internationally renowned ophthalmology journals - the British fournal of Ophthalmology (BFO) and the American fournal of Ophthalmology (AfO).

\section{METHOD AND RESULTS}

Using the public Medline facility provided by the National Institutes of Health, the numbers of prospective studies and case reports published in the $A F O$ and the $B F O$ from January 1980 to December 1999 were determined. These were done using the following keyword searches: "prospective" and "case report." The countries of origin of the articles were counted manually for the years 1990 and 1999 , and were taken as the addresses of the corresponding author. Keyword searching was not possible owing to the non-uniformity of the way the addresses were registered.

The total number of publications remained fairly constant in the $A \mathcal{F O}$ over the two decades (Fig 1A). The percentage of prospective studies increased greatly from $1 \%$ to $12 \%$ (Fig 1B). Case reports, on the other hand, constituted $34-45 \%$ of the published articles (Fig 1C) with no obvious trend.

In comparison, there was a steady increase in the total number of articles (Fig 1A) in the
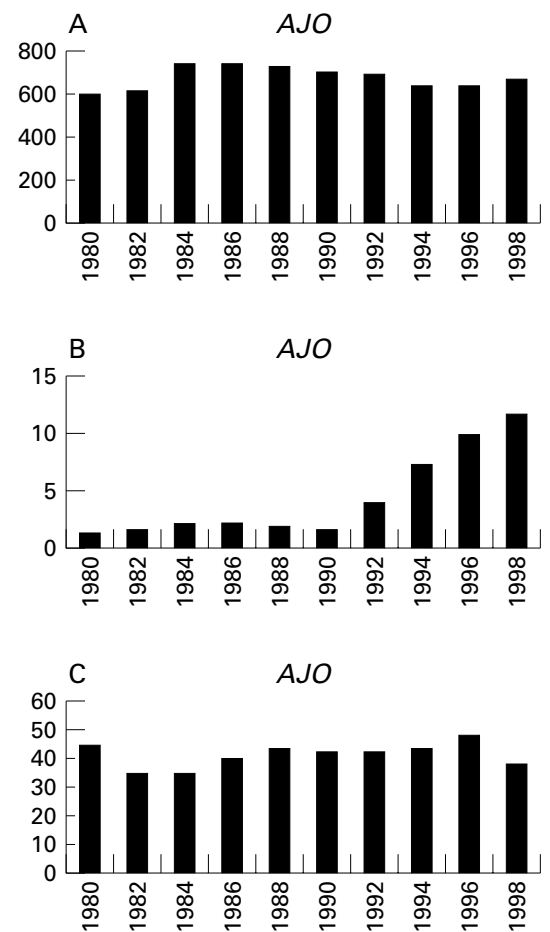

Figure 1 Histograms showing $(A)$ the total number of articles (B) the percentage of prospective articles, and $(C)$ the percentage of case reports in 2 yearly intervals in both journals.
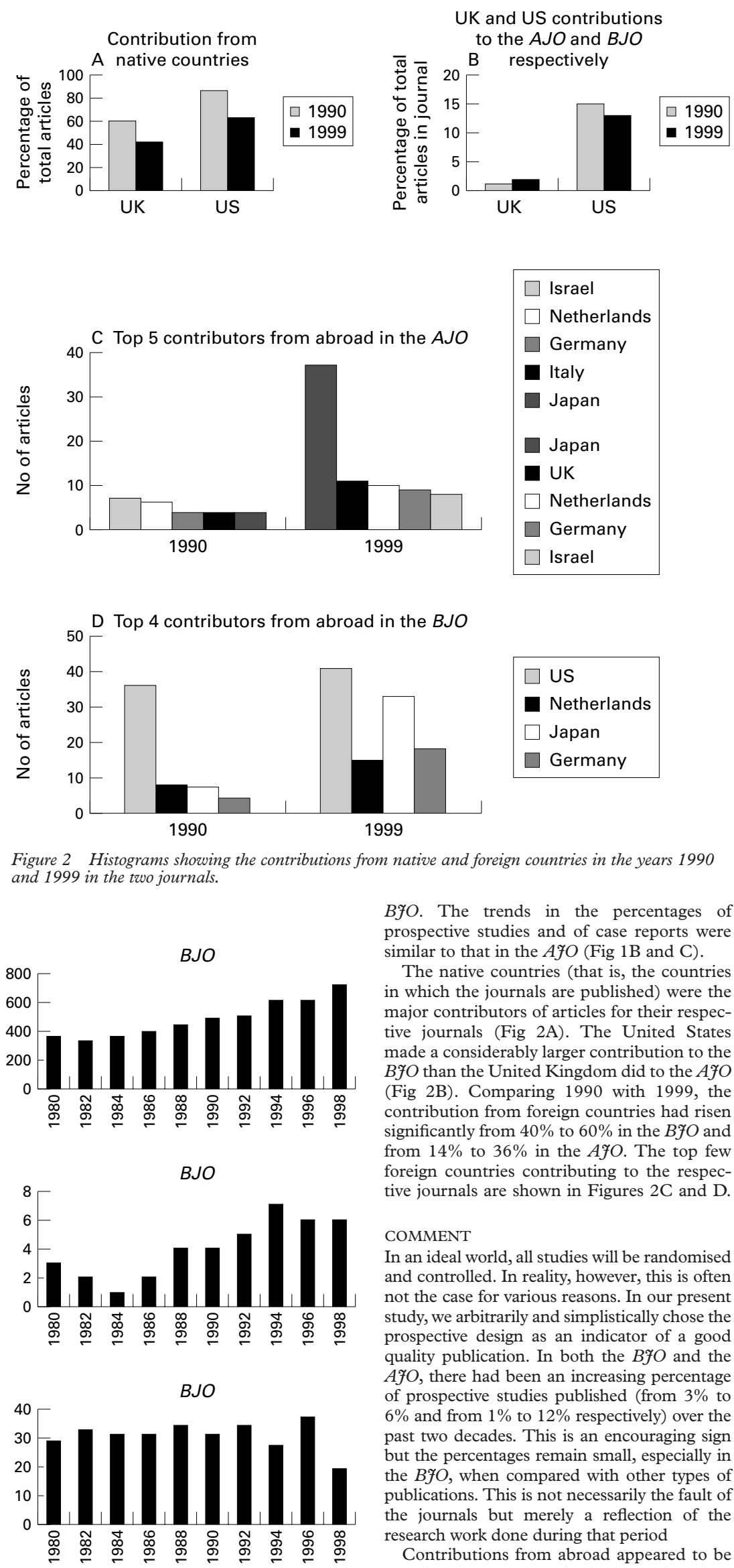

Figure 2 Histograms showing the contributions from native and foreign countries in the years 1990 and 1999 in the two journals.

BFO. The trends in the percentages of prospective studies and of case reports were similar to that in the $A \mathscr{F O}$ (Fig $1 \mathrm{~B}$ and C).

The native countries (that is, the countries in which the journals are published) were the major contributors of articles for their respective journals (Fig 2A). The United States made a considerably larger contribution to the $B \mathcal{O} O$ than the United Kingdom did to the $A \mathcal{F O}$ (Fig 2B). Comparing 1990 with 1999, the contribution from foreign countries had risen significantly from $40 \%$ to $60 \%$ in the $B F O$ and from $14 \%$ to $36 \%$ in the $A F O$. The top few foreign countries contributing to the respective journals are shown in Figures $2 \mathrm{C}$ and D.

COMMENT

In an ideal world, all studies will be randomised and controlled. In reality, however, this is often not the case for various reasons. In our present study, we arbitrarily and simplistically chose the prospective design as an indicator of a good quality publication. In both the $B \mathcal{B O}$ and the $A \mathcal{F O}$, there had been an increasing percentage of prospective studies published (from 3\% to $6 \%$ and from $1 \%$ to $12 \%$ respectively) over the past two decades. This is an encouraging sign but the percentages remain small, especially in the $B \mathcal{F O}$, when compared with other types of publications. This is not necessarily the fault of the journals but merely a reflection of the research work done during that period

Contributions from abroad appeared to be on the increase in both journals when comparing 1990 with 1999 with the BFO 
being the more cosmopolitan of the two. This increasing trend of foreign contribution was also noted by Kaugars et al in the journal Oral Surgery Oral Medicine Oral Pathology.

There are limitations to the present study. The $A \mathcal{F O}$ and the $B \mathcal{F O}$ may not be representative of the international ophthalmic journals from the United States and the United Kingdom respectively. Secondly, the total number of articles may be deceptive as the $B \mathcal{B O}$ and

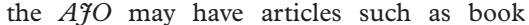
reviews, editorials, letters, etc at different frequencies. Thirdly, there is the possibility of inadequate keyword classification of the publications in the journals. Finally, the address of the corresponding author may not always correspond to the country where the research was performed.

In conclusion, our study suggests that the standard of publications has improved in the $A F O$ and the $B \mathcal{F O}$, with an increasing international contribution over the past two decades.

Proprietary interests: None.

Financial support: None.

Department of Ophthalmology, Ipswich Hospital, Heath Road, Ipswich IP4 5PD, UK

L TONG

A BHAN

Department of Ophthalmology,

Queen's Medical Centre, University Hospital, Nottingham NG7 2UH, UK

Correspondence to: $\mathrm{Mr}$ A Ang, Department of Opthalmology, West Norwich Hospital, Bowthorpe Road, Norwich NR2 3TU

Accepted for publication 22 May 2001

1 Kaugars GE, Riley WT, Grisius TM, et al. Comparison of articles published in Oral Surgery Oral Medicine Oral Pathology in 1972 and 1992. Oral Surg Oral Med Oral Pathol 1994;78:351-3.

\section{BOOK REVIEW}

Colour Atlas of the Eye in Systemic Disease. Eds DH Gold, TA Weingeist. Pp 663; £115. Philadelphia: Lippincott Williams \& Wilkins, 2001. ISBN 0-397-51525-1.

This is a large reference multiauthored, well illustrated text on a multitude of systemic conditions that have ophthalmic manifestations. It certainly is an enjoyable book to "flick through," with some excellent photographs ranging from retinal disorders associated with infection and systemic disease to genetic disorders, including a multitude of pictures on dysmorphic syndrome. There are chapters on relatively rare conditions such as ocular pharyngeal muscular dystrophy, periodic paralysis and myopathies, and encephalopathies associated with vitamin disorders. The list goes on and indeed the book is a useful reference, with illustrations and bullet points on the manifestations seen in these diseases. But, without prior knowledge or other texts to read the book is not easy to use. However, with an impressive 159 chapters every library should have this book is on the shelf. It would offer residents and students from all disciplines the opportunity to appreciate how many diseases have ocular manifestations. What a great subject we are involved with!

A D DICK

\section{NOTICES}

\section{Onchocerciasis}

The latest issue of Community Eye Health (No 38) discusses onchocerciasis and the impact of interventions, with an editorial by Bjorn Thylefors, former director of the Programme for the Prevention of Blindness and Deafness, WHO. For further information please contact Community Eye Health, International Centre for Eye Health, Institute of Ophthalmology, 11-43 Bath Street, London EC1V 9EL. (tel: (+44) (0) 20-7608 6909/6910/6923; fax: (+44) (0) 7250 3207; email: eyeresource@ucl ac.uk) Annual subscription $£ 25$. Free to workers in developing countries.

\section{International Centre for Eye Health}

The International Centre for Eye Health has published a new edition of the Standard List of Medicines, Equipment, Instruments and Optical Supplies (2001) for eye care services in developing countries. It is compiled by the Task Force of the International Agengy for the Prevention of Blindness. Further details: Sue Stevens, International Centre for Eye Health, 11-43 Bath Street, London EC1V 9EL, UK (tel: (+44) (0) 20-7608 6910; email: eyeresource@ucl.ac.uk).

\section{Second Sight}

Second Sight, a UK based charity whose aim are to eliminate the backlog of cataract blind in India by the year 2020 and to establish strong links between Indian and British ophthalmologists, is regularly sending volunteer surgeons to India. Details can be found at the charity website (www.secondsight.org.uk) or by contacting Dr Lucy Mathen (lucymathen@yahoo.com).

\section{SPecific Eye ConditionS (SPECS)}

SPECS is a not for profit organisation acting as an unbrella organisation for support groups of any conditions or syndrome with an integral eye disorder. The SPECS website (www.eyeconditions.org.uk) acts as a portal to support groups, and is a valuable resource for professionals and may also be of interest to people with a visual impairment or who are blind. Further details: Kay Parkinson, SPECS development officer. (tel: +4401803524 238; email: k@eyeconditions.org.uk).

\section{1st St Andrew's Day Festival}

\section{Symposium on Therapeutics}

The 41st St Andrew's Day Festival Symposium on Therapeutics will be held on 6-7 December 2001 at the Royal College of Physicians of Edinburgh. Further details: Ms Eileen Strawn, Symposium Co-ordinator (tel: 01312257324 ; fax: 01312204393 , email: e.strawn@rcpe.ac.uk; website: www.rcpe.ac.uk)

4th International Conference on the Adjuvant Therapy of Malignant Melanoma

The 4th International Conference on the adjuvant therapy of malignant melanoma will be held at The Royal College of Physicians, London on 15-16 March 2002. Further details: Conference Secretariat, CCI Ltd, 2 Palmerston Court, Palmerston Way, London SW8 4AJ, UK (tel: + 44 (0) 207720 0600; fax: + 44 (0) 2077207177 ; email: melanoma@confcomm.co.uk: website: www.confcomm.co.uk/Melanoma).

\section{EUPO 2002 Course Retina}

A course on retina will be held on $15-17$ March 2002 at Erlangen, Germany, where European professors will teach European residents. Further details: Priv Doz Dr Ulrich Schonherr, Friedrich-Alexander-University of Erlangen-Nuemberg, Department of Ophthalmology, Schwabachanlage 6 (Kopfklinikum), D-91054 Erlangen, Germany (tel: +49 9131-853-4379; fax: +49 9131-8534332; email: ulrich-schoenherr@augen.imed. uni-erlangen.de).

\section{XXIXth International Congress of Opshthalmology}

The XXIXth International Congress of Ophthalmology will be held on 21-25 April 2002 in Sydney, Australia. Further details: Congress Secretariat, C/- ICMS Australia Pty Ltd, GPO Box 2609, Sydney, NSW 2001, Australia (tel: +6129241 1478; fax: +612 9251 3552; email: ophthal@icmsaust. com.au; website: www.ophthalmology.aust.com).

\section{International Society for Behçet's}

Disease

The 10th International Congress on Behçet's Disease will be held in Berlin 27-29 June 2002. Further details: Professor Ch Zouboulis (email: zoubbere@zedat.fu-berlin.de).

\section{Singapore National Eye Centre 5th International meeting}

3-5 August 2002, Singapore. Further details: Ms Amy Lim, Organising Secretariat, Singapore National Eye Center, Third Hospital Avenue, Singapore 168751 (tel: +65 322 8374; fax: +65 227 7290; email: amy_lim@ snec.com.sg).

\section{CORRECTIONS}

In a paper published by Minassian et al in the July issue of the BfO (2001;85:822-9) two authors who made significant contributions to the project were omitted. They are Sunny Kaushal, research optometrist, Oxford Eye Hospital, and Nicholas Wingate, research optometrist, Moorfields Eye Hospital. We apologise or this omission.

A translation error occurred in the article by Demailly et al which appeared in the August issue of the BFO (2001;85:921-4). In the abstract (p 921 line 16) and the text (p 922, line 8 ) the dose for carteolol alginate was given as "four times daily" when it should be once daily. We apologise for this error. 